OPEN ACCESS

Edited by:

Octavio Luiz Franco,

Universidade Católica de Brasilia,

Brazil

Reviewed by:

Osmar Nascimento Silva,

Universidade Católica Dom Bosco,

Brazil

Dinesh Sriramulu,

Shres Consultancy (Life Sciences),

India

*Correspondence:

Lothar Lilge

llilge@uhnres.utoronto.ca

Specialty section: This article was submitted to Antimicrobials, Resistance

and Chemotherapy,

a section of the journal

Frontiers in Microbiology

Received: 18 June 2016

Accepted: 29 July 2016

Published: 09 August 2016

Citation:

Fila G, Kasimova K, Arenas Y,

Nakonieczna J, Grinholc M, Bielawski KP and Lilge L (2016) Murine Model Imitating Chronic Wound Infections for Evaluation of Antimicrobial Photodynamic

Therapy Efficacy.

Front. Microbiol. 7:1258.

doi: 10.3389/fmicb.2016.01258

\section{Murine Model Imitating Chronic Wound Infections for Evaluation of Antimicrobial Photodynamic Therapy Efficacy}

\author{
Grzegorz Fila ${ }^{1}, K_{a m o l a}$ Kasimova ${ }^{2}$, Yaxal Arenas ${ }^{3}$, Joanna Nakonieczna', \\ Mariusz Grinholc ${ }^{1}$, Krzysztof P. Bielawski ${ }^{1}$ and Lothar Lilge ${ }^{2,4 *}$
}

\begin{abstract}
1 Laboratory of Molecular Diagnostics, Department of Biotechnology, Intercollegiate Faculty of Biotechnology, University of Gdansk and Medical University of Gdansk, Gdansk, Poland, ${ }^{2}$ Princess Margaret Cancer Centre, University Health Network, Toronto, ON, Canada, ${ }^{3}$ Theralase Inc., Toronto, ON, Canada, ${ }^{4}$ Department of Medical Biophysics, University of Toronto, Toronto, ON, Canada
\end{abstract}

It is generally acknowledged that the age of antibiotics could come to an end, due to their widespread, and inappropriate use. Particularly for chronic wounds alternatives are being thought. Antimicrobial Photodynamic Therapy (APDT) is a potential candidate, and while approved for some indications, such as periodontitis, chronic sinusitis and other niche indications, its use in chronic wounds is not established. To further facilitate the development of APDT in chronic wounds we present an easy to use animal model exhibiting the key hallmarks of chronic wounds, based on full-thickness skin wounds paired with an optically transparent cover. The moistureretaining wound exhibited rapid expansion of pathogen colonies up to 8 days while not jeopardizing the host survival. Use of two bioluminescent pathogens; methicillin resistant Staphylococcus aureus (MRSA) and Pseudomonas aeruginosa permits real time monitoring of the pathogens. The murine model was employed to evaluate the performance of four different photosensitizers as mediators in Photodynamic Therapy. While all four photosensitizers, Rose Bengal, porphyrin TMPyP, New Methylene Blue, and TLD1411 demonstrated good to excellent antimicrobial efficacy in planktonic solutions at 1 to $50 \mu \mathrm{M}$ concentrations, whereas in in vivo the growth delay was limited with 24-48 h delay in pathogen expansion for MRSA, and we noticed longer growth suppression of $P$. aeruginosa with TLD1411 mediated Photodynamic Therapy. The murine model will enable developing new strategies for enhancement of APDT for chronic wound infections.

\footnotetext{
Keywords: photodynamic therapy, topical infection, chronic wounds, bioluminescence, Pseudomonas aeruginosa, methicillin resistant Staphylococcus aureus
}

\section{INTRODUCTION}

Chronic wounds, generally comprising diabetic foot ulcers, pressure ulcers, venous leg ulcers, burn wounds, and wounds "older than 3 months of age" (Kirketerp-Møller et al., 2011) are a worldwide healthcare issue. They are causing a cycle of pain, anxiety and reduced quality of life for the individual patient, and present a considerable cost to the health care providers and patient 
(Dowsett, 2015). It is estimated that chronic wound therapies account for $2-4 \%$ of the total health system expenses, including material cost, nurse time, and hospitalization, that estimate is excluding the indirect costs such as loss of productivity and out-of-pocket expenses for patients (Sen et al., 2009). Over $90 \%$ of chronic wounds contain bacteria and fungi acquired from the skin, oral mucosa, enteric tract, or the environment (Kucera et al., 2014), which prevent tissue remodeling and healing. These bacteria can form a multispecies biofilm, which is often held responsible for the further development of an infection (Kucera et al., 2014). Infections commonly benefit from a priori compromised immune functions due to other diseases and/or their treatments, resulting in the lack of particular immune system components such as B cells, $\mathrm{T}$ cells, antibodies, neutrophils, damage to immune organs etc. Secondary immunodeficiency is often exploited by various microorganisms leading to grievous infections. Immunodeficiency is a well-established risk factor for cancer patients receiving chemotherapy (Pardoll, 2012; Khan et al., 2015).

Any break in the barrier function of the skin and other epithelial layers, predisposes an individual to infections (Schreier and Chatterjee, 2015). One of the major issues in controlling the healing initiation versus infection is sustaining the moisture balance in the tissue. Swollen and suppurative wounds provide perfect growth conditions for anaerobic and aerobic pathogens colonization and expansion thereof (Kirketerp-Møller et al., 2011), whereas a dehydrated wound surface can delay the healing process. Hence, while preserving a moist wound environment is advisable (Lilge et al., 2000) concurrent eradication and suppression of pathogenic microorganisms are highly desirable. A delay or failure in the treatment of moist wounds can lead to bacterial colonization progression and can cause the development of systemic infection.

Currently, there are some therapies to control infected wounds. The most classical - antibiotic therapy - starts to be inefficient as multidrug-resistant strains keep spreading and continue to expand unchecked. The increase in multidrug resistant strains to conventional antibacterial therapies has prompted the development of alternatives antimicrobial therapies, particularly for hard-to-heal wounds (Troxler et al., 2006). Negative pressure wound therapy promotes wound healing by applying a vacuum through a special dressing. It increases the granulation tissue formation and the local blood flow and enhances the bacterial clearance (Ene et al., 2015). Wound dressing saturated with silver, iodine or other antimicrobial agents can help the body achieve the ideal moist, warm healing conditions and simultaneously protect the wound from environmental exposure during this process (Murphy and Evans, 2012). Numerous plants are used in folk medicine against various diseases, albeit the exact mechanism of their action remains largely unknown, although antimicrobial properties of this phytotherapy have been investigated (Halcon and Milkus, 2004; Joubert et al., 2008; Kumar et al., 2008) and many of them are considered biofilm disruption agents. Combination of ethnopharmacology and antibiotic therapy could provide an effective bactericidal tool for the treatment of various bacterial and yeast infections (Taraszkiewicz et al., 2013). Low-Level Laser Therapy (LLLT) seems to exert possibly exploitable antimicrobial effects, especially against Pseudomonas aeruginosa caused infections, whereas it appears to be less or ineffective against other bacterial strains (Nussbaum et al., 2003, 2009).

To address the local nature of chronically infected wounds Antimicrobial Photodynamic Therapy (APDT) provides appealing properties. In APDT, a chemical compound (photosensitizer) absorbs light photons with sufficient quantum energy resulting in its electronic excited state, from which energy can be transferred to biomolecules or to molecular oxygen. The latter leads to the generation of Reactive Oxygen Species (ROS) or singlet oxygen formation, which can cause cell damage and microorganism's death as recently reported by Taraszkiewicz et al. (2013) in in vitro studies of APDT. Localization of the therapy is achieved by two different mechanisms; first the confinement of the activation light to the clinical target area and second the difference in temporal photosensitizer association and uptake by bacteria versus host cells.

While efficacy of APDT has been demonstrated in various planktonic in vitro studies, transition to in vivo studies showed variable results, with some pre-clinical models showing good efficacy, whereas the majority of the studies demonstrated a poor translation of the APDT efficacy in vivo.

Typically, to quantify a therapy's efficacy, based on the eradicated bacterial number, one group of hosts need to be sacrificed immediately after treatment and a second group need to be kept up to $50 \mathrm{~h}$ to determine the microbiota survival fraction. Blood samples can also be taken to analyze possible bacteremia development. Hamblin et al. (2003) and Wong et al. (2005) created full thickness excisional wounds, by using surgical scissors and forceps. Small wounds $(8-9 \mathrm{~mm} \times 12.5 \mathrm{~mm})$ were infected with bioluminescent bacterial strains $P$. aeruginosa $\left(10^{6}\right)$ or Vibrio vulnificus $\left(10^{6}\right)$, allowing observation of wound healing and infection development within individual animals. As wounds remain uncovered, mice had to be kept separate to prevent opportunistic contaminations between animals. Zolfaghari et al. (2009) used two wound models: excisional and superficial. In the first case, shaved and depilated skin was pinched by sterile forceps and a $6 \mathrm{~mm}$ circular area $\left(28 \mathrm{~mm}^{2}\right)$ was cut down till the subcutaneous areolar tissue using scissors. In the second model a $25 \mathrm{~mm}^{2}$ square shaped wound was created by scarification using a $27 \mathrm{G}$ needle. Both skin preparations were inoculated with $10^{8}$ $\mathrm{CFUml}^{-1}$ MRSA cells on the wounds. To assess the effectiveness of the therapy mice were sacrificed upon treatment and the number of surviving bacteria was quantified following incubation on agar plates.

The ideal pre-clinical model of hard-to-heal wounds should be based on an immunocompromised host, thus permitting systematic infection. However, that model requires weeks to be established and carries significant risk of sepsis and survival failure. Also the use of immunocompromised animals can dramatically increase the cost of the research. Minimal animal number, wound drying, dressing changes causing wound oxygenation variations, unnecessary animal pain, or large surface area damage are some of the important factors to be considered during the in vivo experimental design stage. 
Here we demonstrate a Tegaderm ${ }^{\mathrm{TM}}$ based topical infection model for antimicrobial PDT as local antimicrobial therapy, and demonstrate its utility by testing Rose Bengal (RB), porphyrin TMPyP New Methylene Blue (NMB), or TLD1411 mediated APDT.

By the use of strong skin adhering, vapor and light transmitting dressings (Lilge et al., 2000), a moist wound environment is maintained providing perfect condition for wound healing as well as bacterial growth and the development of infection. Dressings can be kept for several days, isolating wounds from environmental- and cross-contaminations, permitting co-housing of animals thus reducing their stress level. Of additional advantage is the use of bioluminescent bacterial strains permitting non-invasive longitudinal monitoring of the therapeutic efficacy in each animal, reducing the number of animals required to achieve statistical significance. Moreover, from an animal care point, the possibility to decrease the wound size as well as provide opportunistic infection prevention will promote better tissue reconstruction.

To demonstrate the utility of the murine model, we validated the efficacy of 4 different photosensitizers RB, porphyrin TMPyP, $\mathrm{NMB}$, and TLD1411 as APDT mediators in planktonic solution, demonstrated the need for a covered wound for maintaining an ongoing infection and finally demonstrated the ability to quantify APDT outcome in this murine model, in vivo as time delay until pathogen regrowth is noted.

\section{MATERIALS AND METHODS}

\section{Animals}

All procedures were approved by the Animal Care Committee (ACC) of University Health Network, Toronto, ON, Canada (protocol number AUP 3303.2). FVB/N mice of both sexes, originally obtained from Xenogen Corporation - Alameda, CA but tested negative for the luciferase gene were used. Animals were housed in 12 -h day/night cycles, at $20^{\circ} \mathrm{C}$ room temperature, granulated food, and water were provided ad libitum.

In total 51 mice were required for this study and randomly assigned to one of 6 groups: (i) non-infected mice $(n=3)$ to establish the duration of normal wound healing. Groups (ii) to (iv) represent infected wounds without APDT comprising: (ii) Tegaderm dressing, (iii) no dressing, and (iv) removal of dressing at the end of day 2 from established infection to evaluate the need for the transparent dressing to maintain the infection control throughout the observation period. For these groups $n=3$ animals were used for each of the two bacterial strains employed here. The APDT treatment groups v) comprised $n \geq 3 \times 2$ bacterial strains for each of the four photosensitizers were tested. Group (vi) evaluated APDT efficacy versus potential LLLT effects employing a light only group for both bacterial strains.

\section{Infected Wound Induction Procedure}

The day before wound establishment, the animals were anesthetized by isoflurane using $4 \%$ for induction and 1.5 to $2.5 \%$ for maintenance delivered via nose cone. Their dorsal surface hair was shaved and remaining hair removed with depilatory lotion. The next day, after repeating anesthesia a pinch of skin in the center of the shaved area $(0.5 \mathrm{~cm}$ to $1 \mathrm{~cm}$ above the ilium bones) was held by tweezers and top layer of the skin is cut off to cause 5 to $6 \mathrm{~mm}$ diameter wound.

Immediately after cutting the skin the wound area was inoculated with $10 \mu \mathrm{l}$ of PBS containing $10^{7} \mathrm{CFU}$ bacteria using a pipette tip. For mice in groups ii to vi Tegaderm ${ }^{\mathrm{TM}}$ was applied immediately as per group assignment and kept for the assigned time.

\section{Dressing}

$1.5 \mathrm{~cm}$ by $1.5 \mathrm{~cm}$ squares of Tegaderm ${ }^{\mathrm{TM}}$ (Transparent Film Roll $3 \mathrm{M}^{\mathrm{TM}}$ ) were cut and applied to the wound area. According to commercial specification, the dressing is breathable permitting oxygen in and moisture vapor out thus allowing the skin to function normally. Tegaderm ${ }^{\mathrm{TM}}$ is transparent and no light attenuation needs to be considered (Lilge et al., 2000). Tegaderm ${ }^{\mathrm{TM}}$ studies have demonstrated positive effect on wound healing process in patients. Patients reported less pain during dressing changes, as well as more comfort with daily usage (Ravenscroft et al., 2006).

\section{Bacterial Strains}

Two bioluminescence pathogens: Methicillin Resistant Staphylococcus aureus (MRSA) strain (XEN) (Caliper, City, State) and Pseudomonas aeruginosa (PAK) were used. PAK is a wild type, commonly studied $P$. aeruginosa strain containing and expressing a full complement of virulence factors. The strain has $\operatorname{lu} x A B$ inserted into its chromosome thereby exhibiting bioluminescent properties (Ramphal et al., 2008).

Bacteria were incubated for $18 \mathrm{~h}$ at $37^{\circ} \mathrm{C}$ in fresh $\mathrm{LB}$ liquid broth. After overnight incubation, tubes were vortexed and the volume adjusted to obtain $10^{7} \mathrm{CFUml}^{-1}$. Next, bacteria were centrifuged and re-suspended in $20 \mu \mathrm{l}$ sterile PBS. To control the concentration of bacteria a serial dilutions of the utilized suspension was prepared and inoculated on fresh LB agar plate followed by incubation for $18 \mathrm{~h}$ at $37^{\circ} \mathrm{C}$. Colonies were counted on the next day to determine the original bacteria concentration.

\section{Light Source}

A custom built LED light source emitting $\lambda=525 \pm 15 \mathrm{~nm}$ (manufactured by Theralase Inc. Toronto, ON, Canada) was employed and the output was focused on the wound area. The light source of non-coherent light, delivering an irradiance of $50 \mathrm{mWcm}^{-2}$ to the wound surface as verified by a NIST traceable optical power meter (Nova Power Meter, Ophir Photonics). The light source was used previously for in vivo phototherapy studies and did not cause thermal effects (Arenas et al., 2013; Fong et al., 2015). The light was administrated continuously for $37 \mathrm{~min}$, resulting in a radiant exposure of $100 \mathrm{Jcm}^{-2}$.

\section{Photosensitizers}

Four Photosensitizers were evaluated for their APDT efficacy in vitro and in vivo: RB, NMB, TMPyP, and TLC1411. All 
photosensitizers were excited at $525 \mathrm{~nm}$ to minimize APDT efficacy differences due to variations in the wavelength dependent penetration depth into the moist infected wounds. Comparing the efficacy between the photosensitizers is thus only a function between their respective molar extinction coefficients at $525 \mathrm{~nm}$.

Rose Bengal (4,5,6,7-tetrachloro-2', $4^{\prime}, 5^{\prime}, 7^{\prime}$-tetraiodofluorescein) (Sigma-Aldrich) is a sodium salt used in medicine as an eye drops to stain damaged conjunctival and corneal cells (Ervin et al., 2010). Maximum absorption wavelength $\lambda_{\max }=548 \mathrm{~nm}$ and the molar extinction coefficient at $525 \mathrm{~nm}$ is $3653 \mathrm{molL}^{-1} \mathrm{~cm}^{-1}$. The singlet oxygen quantum yield was reported as 0.74 (Lee and Rodgers, 1987).

New Methylene Blue is a thiazine class compound used as a dye or antimicrobial agent (Ragas et al., 2013) with an absorption maximum at $\lambda_{\max }=590 \mathrm{~nm}$, a molar extinction coefficient at $525 \mathrm{~nm}$ of $471 \mathrm{molL}^{-1} \mathrm{~cm}^{-1}$ and a singlet oxygen quantum yield of $0.66 \pm 0.04$ in air equilibrated acetonitrile (Ronzani et al., 2013).

TMPyP [5,10,15,20-Tetrakis(1-methyl-4-pyridinio) porphyrin tetra(p-toluenesulfonate)] (Sigma-Aldrich) is a positively charged, two-photon dye, that can bind to DNA in cell cultures and affect the cell viability. Its one photon peak absorption wavelength $\lambda_{\max }=421 \mathrm{~nm}$. Its molar extinction coefficient at $525 \mathrm{~nm}$ is $1306 \mathrm{molL}^{-1} \mathrm{~cm}^{-1}$, and it has a singlet oxygen quantum yield of 0.74 (Reddi et al., 2002).

TLD1411 is a $\mathrm{Ru}(\mathrm{II})$ coordination complex of the form $\left[\mathrm{Ru}\left(2,2^{\prime}\right.\right.$ -bipyridine $) 2\left(2-\left(2^{\prime}, 2^{\prime \prime}: 5^{\prime \prime}, 2^{\prime \prime \prime}\right.\right.$-terthiophene $)$-imidazo[ $[4,5-\mathrm{f}][1,10]$ phenanthroline) $] 2+$, originally synthesized by Dr. McFarland at Arcadia University and provided by Sigma Aldrich Fine Chemicals (Milwaukee, WI, USA). Its use as an APDT agent has been previously described (Arenas et al., 2013). Its absorption maximum is $430 \mathrm{~nm}$ in water and its molar extinction coefficient at $525 \mathrm{~nm}$ is $831 \mathrm{molL}^{-1} \mathrm{~cm}^{-1}$. The singlet oxygen quantum yield was reported as $>0.9$ in complete media (Shi et al., 2015).

Figure 1 shows the wavelength resolved molar extinction coefficient of these four photosensitizers.

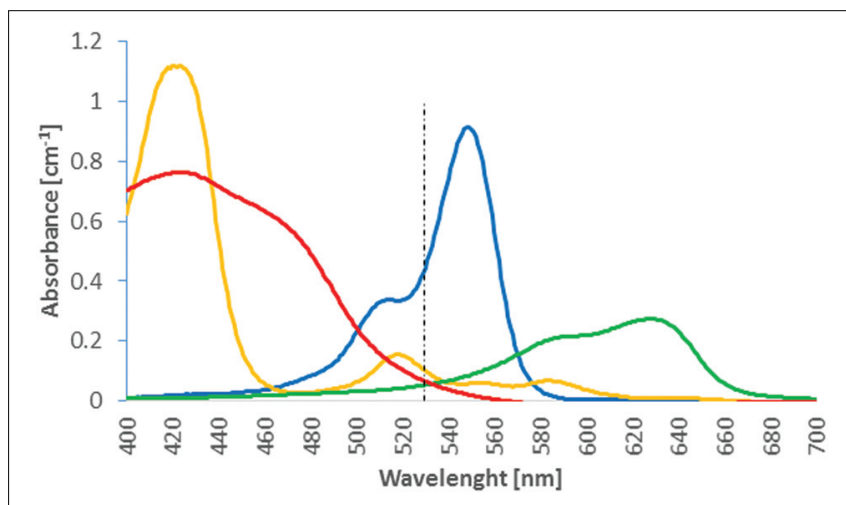

FIGURE 1 | Absorption coefficient of $10 \mu \mathrm{M}$ solutions of Rose Bengal (blue), New Methylene blue (NMB) (green), TMPyP (orange), and TLD1411 (red) as function of wavelength. The $525 \mathrm{~nm}$ Antimicrobial Photodynamic Therapy (APDT) activation wavelength is indicated.
All photosensitizers were freshly prepared prior to the procedure as $1 \mathrm{mM}$ stock solution in water, and diluted to the proper test concentration before being added to the bacterial culture.

\section{In vitro Experiments}

All procedures were performed using sterile 96-well plates. Bacteria were diluted in fresh Luria-Bertani (LB) broth to a final planktonic concentration of $3 \cdot 10^{7} \mathrm{CFUml}^{-1}$. The photosensitizer solution was added at predetermined concentrations ranging from $1 \mu \mathrm{M}$ to $150 \mu \mathrm{M}$ equivalent to a final concentration of $2 \cdot 10^{8}$ to $3 \cdot 10^{10}$ PS molecules per bacterium. $100 \mu \mathrm{l}$ of the PS solution was pipetted into wells followed immediately by photoactivation using the light source described above. Non-irradiated sample served as negative controls. Upon completion of light administration, samples were serial diluted and inoculated on fresh LB agar plates. A positive result required more than 3 magnitudes of $\log _{10}$ per $\mathrm{CFUml}^{-1}$ reduction in these planktonic conditions. PDT irradiation used a 96-diode laser array delivering $100 \mathrm{Jcm}^{-2}$ total radiant exposure.

\section{In vivo Experiments}

All procedures of wound establishment and bacterial inoculation were executed as described above. $30 \mathrm{~min}$ post bacterial inoculation $30 \mu \mathrm{l}$ of the $500 \mu \mathrm{M}$ photosensitizer solutions was injected under the dressing by Hamilton syringe and allowed to spread over the wound for an estimated final concentration of $\sim 2 \cdot 10^{9}$ photosensitizer molecules per bacterium. Photoactivation was initiated after $30 \mathrm{~min}$ of wait period.

\section{Bioluminescence Imaging}

Images were collected using the IVIS Spectrum imaging system (Caliper Life Sciences, Hopkinton, MA, USA). For imaging, mice were anesthetized using isoflurane, as described above. Mice were imaged at specific time points: immediately after bacteria inoculation, prior to and after PS injection, prior to and after light administration, then every $10 \mathrm{~min}$ post APDT therapy up to $2-3 \mathrm{~h}$, followed then daily up to 5 th day or the endpoint determined in the protocols. APDT efficacy for bacterial inactivation was measured by loss of bioluminescent signal, defined as average radiance $\left[\mathrm{hus}^{-1} \mathrm{~cm}^{-2} \mathrm{sr}^{-1}\right]$ and subjective evaluation of the wound's appearance. The bioluminescence (BLI) radiance was determined by selecting equal size of the region-of-interest (ROI) directly over the wound and between the two shoulders blade to determine the cameras dark counts.

\section{RESULTS}

\section{Infection Development in Open versus Covered Wounds}

In Tegaderm ${ }^{\mathrm{TM}}$ covered wounds an increase in bioluminescent signal was observed after inoculation with either PAK or XEN strain, starting from day 0 up to 3rd (PAK) or 4th (XEN) day of experiment. Then, a plateau phase was reached lasting up to 

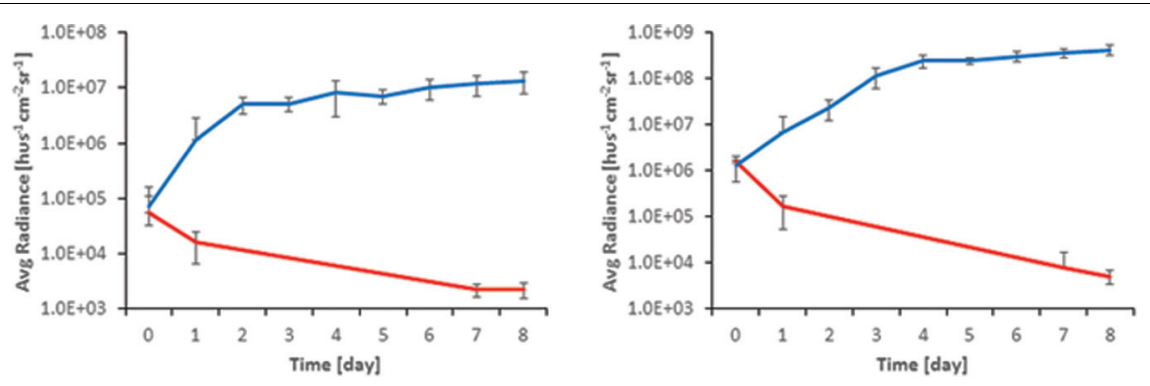

FIGURE 2 | Comparison of BLI signal from open (red) and Tegaderm ${ }^{\mathrm{TM}}$ dressed (blue) wounds for (Left) XEN and (Right) PAK.

8th day after wound inoculation with bacteria (Figure 2). On the contrary, in wounds left open from the beginning as well as those where the Tegaderm ${ }^{\mathrm{TM}}$ was removed after $48 \mathrm{~h}$, a reversal of the initial increase in BLI radiance was observed. The wound's appearance was dry after 2 days, and a pink appearance was retained.

The appearance of the Tegaderm ${ }^{\mathrm{TM}}$ dressed wound changed from the clear raw appearance to yellowish dense mucus on day 2 and 3, whereas they appeared yellow for the remainder of the observed duration. Often the wound expanded and the infection bulged the dressing outward as shown in Figure 3, for PAK inoculated wounds.

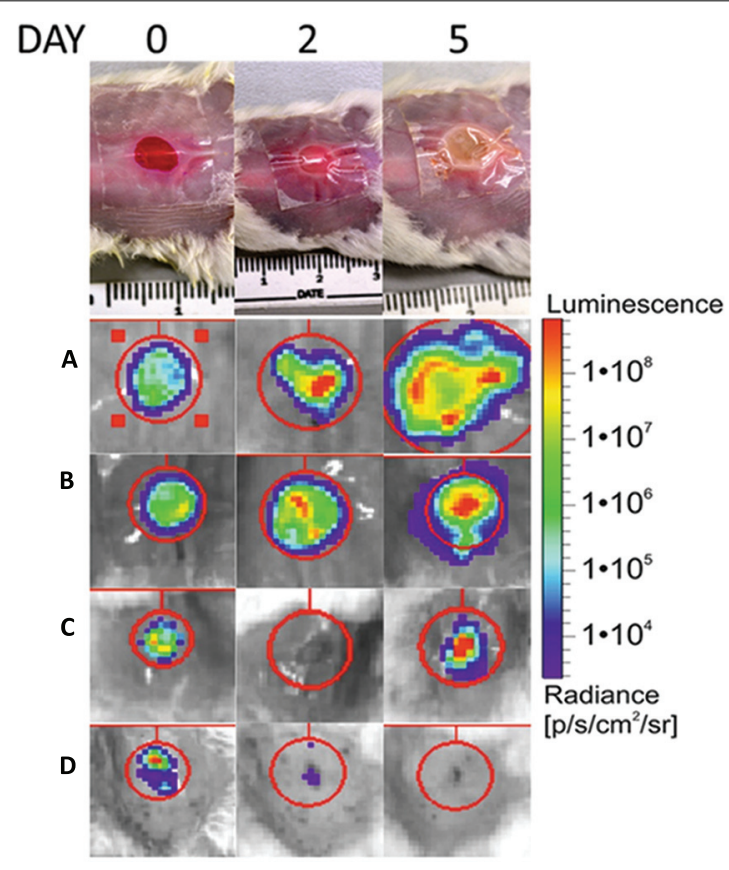

FIGURE 3 | Top shows the visual appearance of the wound over the 5-day observation period for a covered; non-treated wounds (A), covered, treated with Rose Bengal, Pseudomonas aeruginosa infected wound (B); covered; treated with TLD1411; Staphylococcus aureus infected wounds (C); uncovered, non-treated; infected wounds (D). The spread of the infection is clearly visible in (A and $\mathbf{B})$, while (C) showed delay of infection. Left upper corners: Shown are examples of BLI signal as function of time over the first 5 days following wound generation and infection.

\section{APDT in Planktonic Solutions}

There was no significant dark toxicity of the photosensitizers toward the investigated bacteria within the concentration range studied (up to $500 \mu \mathrm{M}$ ), except for TLD1411 at > $10 \mu \mathrm{M}$ for XEN. Whereas, upon illumination all studied photosensitizers showed activity against PAK, and TLD1411 also against XEN bacteria; see Figures 4 and 5, for PAK and XEN results, respectively. The susceptibilities of bacteria to APDT mediated by one of the four sensitizers, was tested at increasing concentration of photosensitizers at three radiant-exposures ranging from 50 to $150 \mathrm{Jcm}^{-2}$.

Rose Bengal showed positive effect toward PAK with $75 \mu \mathrm{M}$ concentration and $50 \mathrm{Jcm}^{-2}$ radiant exposure. Increasing light dose up to $150 \mathrm{Jcm}^{-2}$ resulted in a decreasing $\mathrm{RB}$ concentration required to elicit a response. $50 \mu \mathrm{M}$ of $\mathrm{RB}$ and $150 \mathrm{Jcm}^{-2}$ decreased below the level of detection CFU ml ${ }^{-1}$ units up to 6 $\log _{10}$ (Figure 4A). APDT response reciprocity between radiant exposure and $\mathrm{RB}$ concentration breaks down for concentration above $50 \mu \mathrm{M}$ presumably due to self-shielding within the $1 \mathrm{~cm}$ path length in the planktonic solutions used in these experiments, see below.

New Methylene Blue showed least antimicrobial activity against PAK. Only $10 \mu \mathrm{M}$ concentration showed reduction of $3 \log _{10}$ of survival cells when $100 \mathrm{Jcm}^{-2}$ were applied, whereas $50 \mathrm{Jcm}^{-2}$ failed to elicit a beneficial APDT response, both presumably due to self-shielding. At higher doses the photosensitizer showed no significant impact on efficacy. Unlike Rose Bengal, NMB had no dose-dependent efficacy, thus no significant difference in PAK inactivation from 10 to $150 \mu \mathrm{M}$ when exposed the solution to $150 \mathrm{Jcm}^{-2}$ (Figure $4 \mathrm{~B}$ ).

Porphyrin TMPyP reached a positive anti-bacterial effect against PAK when 150 or $100 \mathrm{Jcm}^{-2}$ were administered. Increasing the light exposure led to a higher inactivation effect at lower PS doses and even $10 \mu \mathrm{M}$ concentration exhibit significant CFU $\mathrm{ml}^{-1}$ reduction. The combination of $75 \mu \mathrm{M}$ concentration and $150 \mathrm{Jcm}^{-2}$ of green light resulted in $6 \log _{10}$ eradication of PAK (Figure 4C). As for NMB $50 \mathrm{Jcm}^{-2}$ did not initiate sufficient PAK inactivation again due to self-shielding within the $1 \mathrm{~cm}$ path length cuvette. Self-shielding also caused loss of reciprocity between light and PS doses.

TLD1411 was tested only for $100 \mathrm{Jcm}^{-2}$ against PAK, so more than $6 \log _{10}$ was achieved at the lowest concentration of $1 \mu \mathrm{M}$ (Figure 4D) effectively reaching sterilization levels. 

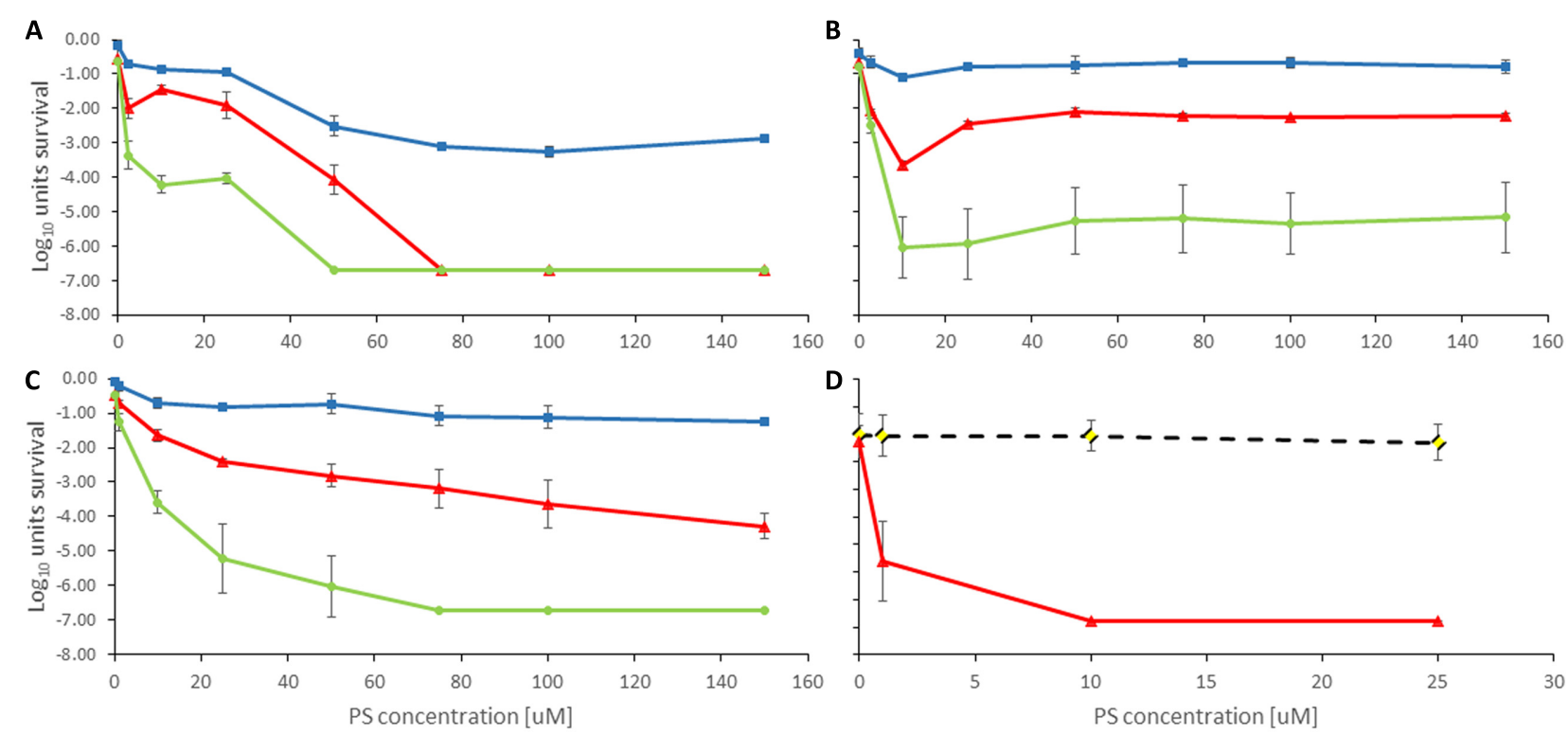

FIGURE 4 | Antimicrobial Photodynamic Therapy inactivation in planktonic solutions for PAK with (A) Rose Bengal, (B) NMB, (C) TMPyP, and (D) TLD1411. The radiant exposure used is indicated as blue squares $=50 \mathrm{Jcm}^{-2}$, red triangles $=100 \mathrm{Jcm}^{-2}$, and green circles $=150 \mathrm{Jcm}^{-2}$. The black dotted line with rhombus in (D) indicates dark toxicity.

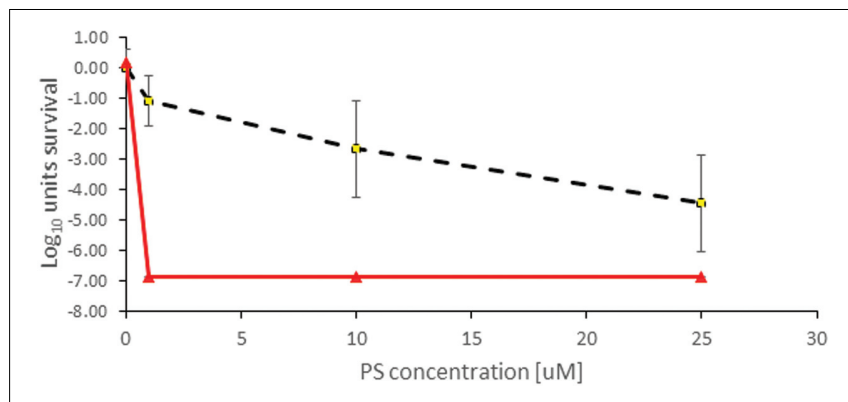

FIGURE 5 | Antimicrobial Photodynamic Therapy inactivation in planktonic solutions for XEN mediated by TLD1411. The employed radiant exposure is indicated as black dotted line with rhombus $=0 \mathrm{Jcm}^{-2}$, red triangles $=100 \mathrm{Jcm}^{-2}$.

Only TLD 1411 was tested for its activity against XEN in these studies, and similar to PAK, $6 \log _{10}$ inactivation was achieved with the lowest concentration of $1 \mu \mathrm{M}$ (Figure 3). However, while no significant dark toxicity against PAK was observed, TLD1411 showed significant dark toxicity, $\sim 3 \log _{10}$, at $10 \mu \mathrm{M}$ against XEN as noted already above.

Table 1 shows the critical concentration required to achieve 3 $\log _{10}$ and $6 \log _{10}$ reduction in bacterial cell count for the highest radiant exposure tested at $525 \mathrm{~nm}$.

\section{In vivo APDT in Infected Wounds}

Unlike for in vitro planktonic cultures, the in vivo response was much more moderate, with none of the photosensitizers achieving sterilization of the wound equivalent to a $6 \log _{10}$
TABLE 1 | Phototoxic sensitizer concentrations.

\begin{tabular}{lccccc}
\hline \multirow{2}{*}{ Photosensitizer } & \multicolumn{2}{c}{ PAK } & & \multicolumn{2}{c}{ XEN } \\
\cline { 2 - 3 } \cline { 5 - 6 } & $3 \log _{10}$ & $\mathbf{6} \log _{10}$ & & $3 \log _{10}$ & $\mathbf{6} \log _{10}$ \\
\hline Rose Bengal & $10 \mu \mathrm{M}$ & $50 \mu \mathrm{M}$ & & $\mathrm{N} / \mathrm{A}$ & $\mathrm{N} / \mathrm{A}$ \\
NMB & $10 \mu \mathrm{M}$ & $>150 \mu \mathrm{M}$ & & $\mathrm{N} / \mathrm{A}$ & $\mathrm{N} / \mathrm{A}$ \\
TMPyP & $10 \mu \mathrm{M}$ & $75 \mu \mathrm{M}$ & & $\mathrm{N} / \mathrm{A}$ & $\mathrm{N} / \mathrm{A}$ \\
TLD1411 & $1 \mu \mathrm{M}$ & $10 \mu \mathrm{M}$ & & $<1 \mu \mathrm{M}$ & $<1 \mu \mathrm{M}$ \\
\hline
\end{tabular}

reduction. In fact, not even clearing at $3 \log _{10}$ was attainable based on BLI radiance measurements, see Figure 6.

As there was a variation in the group average BLI radiance for PAK studies, the BLI signal was normalized at $t=0$ just prior to PS administration, permitting comparison of the APDT effects.

Untreated wounds with expanding bacterial counts reached bioluminescence signal saturation at the first day. The two principal reasons for this saturation effect are increased infection thickness reabsorbing the bioluminescence photons and increased light scattering from the wound further confining the $\mathrm{BLI}$ radiance to the infection.

Rose Bengal mediated APDT resulted in the least BLI radiance reduction, $\sim$ one order of magnitude, or $\sim 3.2$ pathogen division times, presenting limited benefit to wound management. NMB had the highest BLI radiance reduction following APDT $(\sim$ two orders of magnitude); however, the BLI radiance regain was faster than in untreated wound suggesting that the radiance loss was due to biochemical luminescence quenching or bleaching rather than bacterial inactivation. TMPyP had the 2 nd best APDT induced BLI radiance reduction, whereas post APDT signal gains were comparable to untreated wounds suggesting 


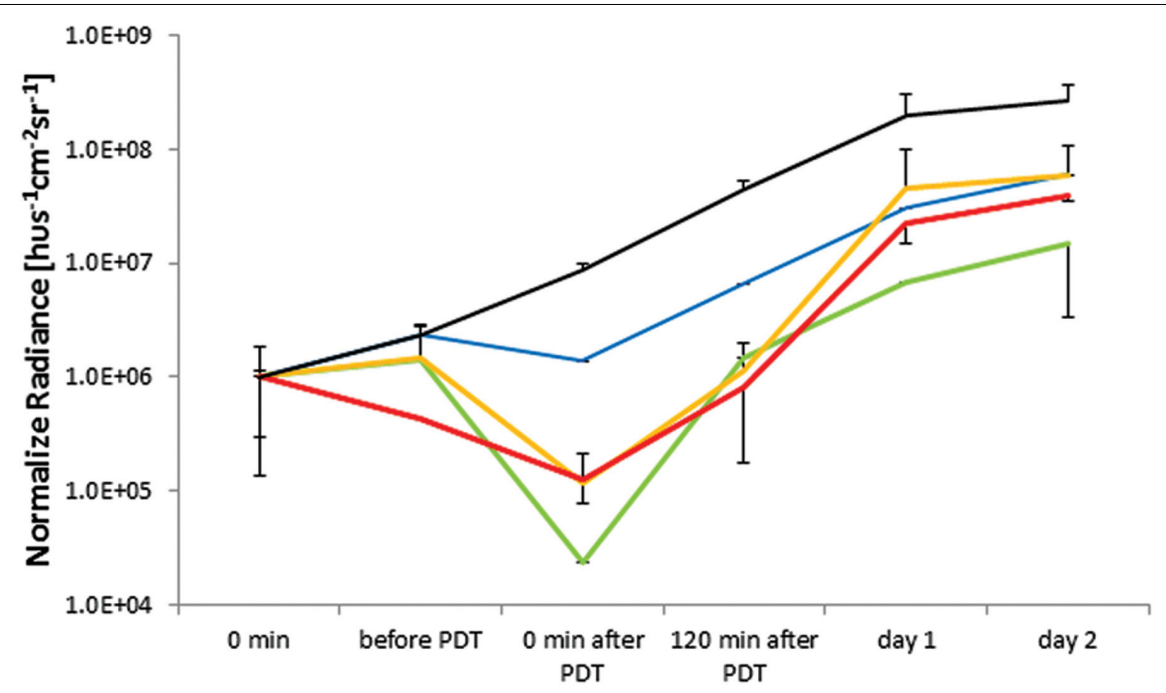

FIGURE 6 | In vivo APDT bioluminescence for PAK in an untreated control (solid black), Rose Bengal (blue), NMB (green), TMPyP (orange), and TLD1411 (red). The concentration of either PS was $\sim 2 \cdot 10^{9}$ molecules per bacterium.

true PAK inactivation or destruction by APDT. TLD1411 showed signs of dark toxicity prior to PDT with accelerated BLI radiance loss during PDT. BLI radiance recovery over the first $2 \mathrm{~h}$ was slower and accelerated over the next $22 \mathrm{~h}$, reaching BLI radiance increase rate comparable to untreated control wounds.

Nevertheless, it should be noted that APDT mediated by either photosensitizer resulted in a lower BLI radiance intensity than non-treated control at $120 \mathrm{~min}$, demonstrating the ability to delay PAK infection progression by APDT of induced wounds, see Figure 6.

The temporal evaluation of the XEN associated BLI in untreated controls and TLD1411 mediated APDT is shown in Figure 7, whereby untreated wounds reached BLI signal saturation at 2 days post infection. The TLD1411 concentration per XEN bacterium is estimated at $\sim 2 \cdot 10^{7}$ and photo-irradiation showed a continuous decrease in BLI radiance up to $90 \mathrm{~min}$ post PDT (120 min time point). The regain of BLI radiance was not significant until day 4 , followed by a signal increase rate comparable to the untreated control.

Group vi, included for evaluation of APDT efficacy versus potential LLLT stimulatory effects due to light irradiation with both bacterial strains were identical to group ii (non-treated, infected wounds covered by Tegaderm). Bioluminescence radiance signal increased for 2 days, reaching $10^{8}$ and $10^{6} \mathrm{hus}^{-1} \mathrm{~cm}^{-2} \mathrm{sr}^{-1}$ of average normalized radiance for PAK and $\mathrm{XEN}$, respectively, and remained constant for the remainder of the observation period (data not shown). Hence, LLLT does not appear to be a confounder to PAK or XEN survival at $525 \mathrm{~nm}$.

\section{DISCUSSION}

While various infected wound models have been published, there is still a need for an applicable model mimicking the clinical features of the disease, with short implementation time and efficient translation of the study results into clinical practice. Lee et al. (2011) created a large wound (1.5 by $2.5 \mathrm{~cm}$ ) by tape-stripping and sandpaper based removing of stratum corneum and inoculating a bacterial suspension containing $\sim 10^{10} \mathrm{CFUml}^{-1} \mathrm{~S}$. aureus cells. Biopsies were collected at specific time points to monitor wound healing and host cell growth. Mice were kept in individual cages to prevent cross contamination, affecting overall well-being of the animal by causing stress. Saito et al. (2012) exposed dorsal skin to $90^{\circ} \mathrm{C}$ to induce burn injury up to $20 \%$ of the total body surface area and then inoculated with $10^{8} \mathrm{CFU}$ of $P$. aeruginosa. The model mimics large, festering wounds and can cause high pain and stress levels. Application of an occlusive dressing to avoid cross contamination is favorable in this model. Similar wounds were established by Hashimoto et al. (2012), where preheated steel was applied to the dorsal surface. Large burned areas (6$6.5 \mathrm{~cm}^{2}$ ) were immediately infected using $10^{8} P$. aeruginosa cells.

The murine model of an infected wound presented here is robust and spontaneous bacterial clearing was not observed in the Tegaderm ${ }^{\mathrm{TM}}$ covered wounds, thus it presents a favorable environment for testing APDT efficacy in vivo. The wound cover allows for oxygen and water vapor across the barrier and does not impede light penetration either for BLI, APDT, or LLLT (Lilge et al., 2000). It provides constant growth environment for both PAK and XEN, as demonstrated in Figures 2 and 3, but prevents opportunistic and environmental wound cross-contamination. Several mice can be housed in the same cage reducing stress levels during the experiment. Wounds, which were not covered or intentionally uncovered, dried out rapidly and infections were controlled without intervention either by the murine immune system or simple dying of the bacterium in an unfavorable environment possibly leading to an overestimation of the APDT efficacy. 


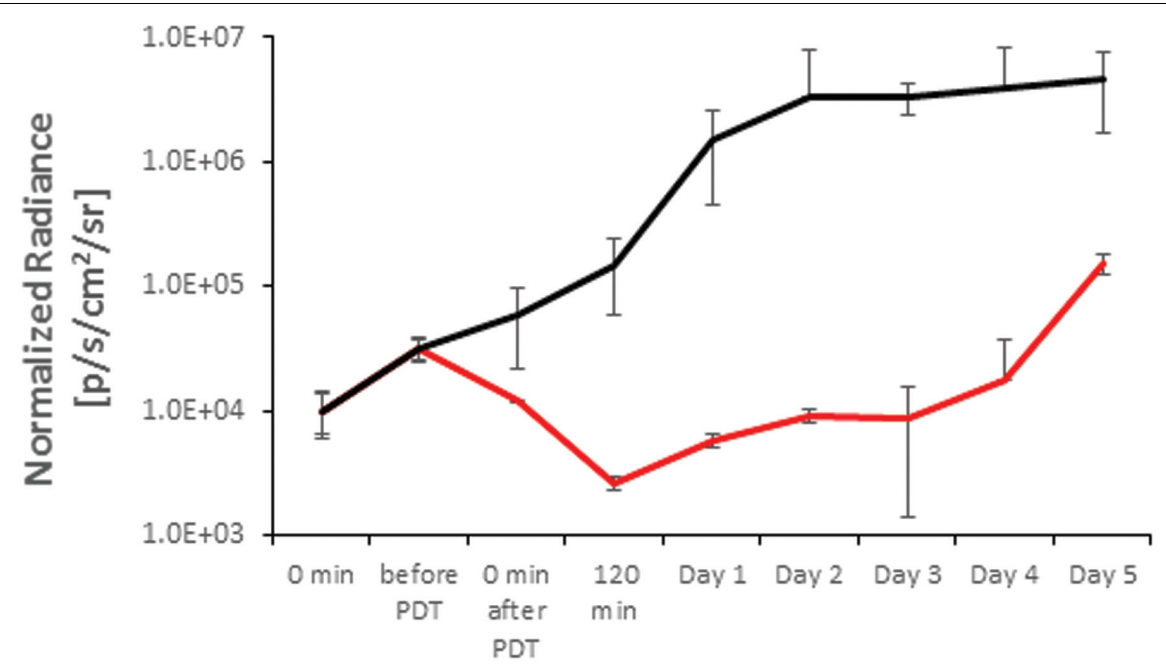

FIGURE 7 | In vivo modulation of the XEN bioluminescence in an untreated control (solid black) and by TLD1411 mediated APDT (red).

Here the model was only utilized in the acute stage of infection, so sufficiently long to open the possibility of resilience against APDT, but insufficient to generate a deep infection, particularly because the $\mathrm{pO}_{2}$ in chronically infected wounds has been reported to be $30 \%$ to $90 \%$ lower than in healthy tissue (Schreml et al., 2010). The latter may require photosensitizers exploiting both type I and II APDT mechanisms (Fong et al., 2015; Shi et al., 2015).

However, the combination of BLI together with consistent growth environment allows testing various APDT protocols including the number of retreatment frequencies and extending the infection control.

As the APDT activation wavelength impacts the penetration depth in the in vivo model, the most common wavelength of $525 \mathrm{~nm}$ was selected. However, the varying molar extinction coefficient of these 4 photosensitizers led to self-shielding and possibly with that reason limited efficacy in planktonic solution for RB and NMB was observed. Selecting different concentrations to match the molar extinction coefficient would impact on the number of photosensitizers per bacterium, whereas selecting different wavelength to match the molar extinction coefficient at constant concentration would affect the light distribution in the tissue and hence by diffuse reflected light also the photon density at the tissue surface.

At time of APDT light treatment in vivo the target must be considered 2D with minimum full-thickness wounds and no observable presence of blood in an infected area. Hence, comparing the efficacy using this animal model system is valid purely on tissue optics consideration.

Nevertheless, the added attenuation of PS in in vivo condition needs to be considered, particularly when comparing PS efficacy in APDT. The worst-case impact on APDT radiance exposure on the target can be estimated based on the solution volume (30 $\mu \mathrm{l}$ ) over the $6 \mathrm{~mm}$ diameter wound, which would result in an $\sim 1 \mathrm{~mm}$ layer large compared to the diffusion distance of the ROS. As the PS acts also as a $525 \mathrm{~nm}$ light filter prior to the pathogens on the tissue surface, the resulting optical densities are $\mathrm{RB}=1.83, \mathrm{NMB}=0.235, \mathrm{TMPyP}=0.653$, and TLD1411 $=0.4155$, respectively. In particular, for $\mathrm{RB}$ the high concentration employed in conjunction with the high molar extinction coefficient eventually caused a reduction of the radiant exposure at the microbes to $1.48 \mathrm{Jcm}^{-2}$. Similarly, the worst-case radiant exposures for the other photosensitizers would be $\mathrm{NMB}=58.2 \mathrm{Jcm}^{-2}$, TMPyP $=22.2 \mathrm{Jcm}^{-2}$, and TLD1411 $=38.4 \mathrm{Jcm}^{-2}$, respectively. However, the solution was rapidly absorbed into the tissue and the filter effect by the photosensitizer had a lesser impact on the sensitizer specific APDT efficacy differences.

Conversely, trying to achieve a comparable OD in the tissue to eliminate effect of self-shielding as a confounding factor. There is a need for either adjustment of the photosensitizer's concentration affecting the ratio of photosensitizing molecules per bacterium or selecting particular wavelength for each photosensitizer so that the molar extinction coefficients are the same, which in turn would impact the photon distribution in the tissue. Hence, our approach of using constant wavelength and radiant exposures in the sensitizer was compared.

To maintain reasonable penetration of the activated light into the tissue, the presence of oxy- or deoxy-hemoglobin needs to be considered as their absorption coefficients for $525 \mathrm{~nm}$ are $>10^{2} \mathrm{~cm}^{-1}$ and the penetration depth would be limited to $<100 \mu \mathrm{m}$ (Jacques, 2013). Hence, the model can only be valid for wounds free of blood or scab. When treating burns a longer absorption wavelength is required for NMB or mixture of $\mathrm{Ru}(\mathrm{II})$ containing photosensitizer with carrying molecules, such as transferrin, which notably increases their molar extinction coefficient in the 600 to $800 \mathrm{~nm}$ range (Kaspler et al., 2016). Care must be taken regarding possible LLLT effects at these longer wavelength (Nussbaum et al., 2003).

A radiant exposure of $150 \mathrm{Jcm}^{-2}$ can be achieved in $12.5 \mathrm{~min}$ according to the guidelines for safe irradiation of the skin, and the duration is also clinically acceptable. However, the experiments 
showed that for optimal efficacy of the therapy, a maximum irradiance of $50 \mathrm{mWcm}^{-2}$ should not be exceeded allowing sufficient oxygen diffusion throughout the Tegaderm ${ }^{\mathrm{TM}}$ wound cover. So care must be taken as possible photosensitizer reuptake into mammalian host cells, particular cells associated with the immune system needs to be considered when the irradiation times need to be extended significantly.

The efficacy of various photosensitizers against bacteria in planktonic solutions have been previously published for RB (Melo et al., 2011) and TMPyP (Cassidy et al., 2010) reporting concentration of $10 \mu \mathrm{mol} / \mathrm{L}$ (6 log of killing) and $250 \mu \mathrm{g} / \mathrm{L} \mathrm{(4} \mathrm{log}$ of killing), respectively.

All photosensitizers have displayed good to excellent efficacy in bacterial inactivation for planktonic solutions particularly for PAK where 3 of 4 reached $6 \log _{10}$ inactivation which is considered as sterilization state and the other photosensitizer (NMB) achieved at least $3 \log _{10}$ of inactivation for $150 \mathrm{Jcm}^{-2}$. The efficacy limits for RB and TMPyP are probably an artifact due to their high molar extinction coefficient and the high concentration used, which prevented effective illumination of the planktonic solution over the $1 \mathrm{~cm}$ sample depth. Radiant exposure with a much lower absorption coefficient of NMB compared to $\mathrm{RB}$, its limited efficacy cannot be attributed to self-shielding. Additionally, as the photosensitizers have comparable singlet oxygen quantum yield one needs to conclude that NMB does not associate well with PAK. TLD1411 appears to present higher efficacy compared to other photosensitizers with a concentration less than $10 \mu \mathrm{M}$ being sufficient for transient PAK sterilization at $100 \mathrm{Jcm}^{-2}$. This may be explained by the ability of these ruthenium complexes to lead to DNA cleavage (Mari et al., 2014; Shi et al., 2015).

The high efficacy of these PSs in vitro is poorly translated into the in vivo applications, as previously noted (Amin et al., 2016). The reason for this efficacy loss can be explained by host factors as well as bacterial burden and the virulence of individual strains as previously noted (Hamblin et al., 2003) and reflects a more challenging environment also for the treatment of human superficial infections.

One caveat to these experiments is the small number $(n=4-5)$ employed for the different study groups. While this sample size is too small to allow statistical analysis of the results between photosensitizers, it is large enough to demonstrate an APDT effect and the report time line for BLI signal delay and time to signal saturation.

While only up to a $1.5 \log _{10}$ reduction in BLI associated radiance was detected by $\mathrm{PS}$ mediated APDT in vivo, it may still be of clinical use. Based on the BLI radiance expression of the PAK and XEN growth curve, pre and post light exposure, it becomes evident that for certain protocols and conditions APDT treated wounds do not reach the BLI radiance intensity of the unexposed control for at least $24 \mathrm{~h}$ in case of PAK mediated infection and more than 4 days for XEN mediated ones. Despite the lack of complete response in wound sterilization this additional time increases the period to early onset of risk for local or systemic infection, thus providing the possibility to perform wound culture and antibiotic sensitivity testing, as about $90 \%$ of blood and other cultures are detected and identified within $48 \mathrm{~h}$ (Cockerill et al., 2004). Peptide nucleic acid fluorescence in situ hybridization (PNA FISH) methods are currently used for direct identification of selected Staphylococcus, Enterococcus, Klebsiella, and Candida species in less than $2 \mathrm{~h}$ (Shepard et al., 2008). While a 1 to $2 \mathrm{~h}$ delay for PCA determination of loss or the presence of genes encoding antibiotic resistance are available and appear reasonable, PCA will not describe functional protein and may be misleading, hence, generally antibiotic susceptibility testing commonly requires 1 to 3 days and hence a general infection control for 24 to $72 \mathrm{~h}$ is desirable.

\section{CONCLUSION}

Our murine model of consistent local wound infection with Staphylococcus aureus (MRSA) and P. aeruginosa was similar to chronic wounds, without spontaneous clearing due to wound drying. The progress of the infection can be monitored realtime for bioluminescent bacteria, thus permitting the rapid evaluation of APDT agents and treatment protocols in vivo under physiologically relevant conditions for bacterial growth.

The in vivo evaluation of antimicrobial effect of photosensitizers responded less effectively compared to what were seen in vitro, an observation also reported by others; however, the potentially significant clinical reduction of the bacterial load remains apparently feasible, causing delay in full activation of inflammatory response and hence reducing the risk of developing sepsis.

The presented murine model is a suitable platform to evaluate the feasibility and safety of repeated cycles of PDT, based on clearance time of photosensitizer from host mammalian cells.

\section{AUTHOR CONTRIBUTIONS}

GF has performed in vitro experiments with the use of $\mathrm{RB}$, TMPyP, and NMB against $P$. aeruginosa, as well as all in vivo procedures. He wrote the first draft of the manuscript. KK assisted and supported the in vivo experiments. YA performed the in vitro experiments. JN: participated in the conception and design of the work; advised on the experimental design and writing of the manuscript. MG participated in the conception and design of the work; drafted the manuscript and revised it critically for important intellectual content. KB participated in designing of the experiments and helped drafting manuscript. LL designed the experiments together with Drs. MG, JN and $\mathrm{KB}$, supervised $\mathrm{GF}, \mathrm{KK}$, and $\mathrm{YA}$ on a daily basis and is the senior responsible author for the manuscript together with GF. He also acts as the corresponding author on this manuscript.

\section{FUNDING}

The authors acknowledge financial support through the Ontario Ministry of Health and Long Term Care (LL). GF 
acknowledges financial support through the European Social Fund: Human Capital" National Cohesion Strategy, as well as NCBiR Project LIDER, Nanobiotechnology as an innovative approach to burn wound infection treatment" nr LIDER/32/36/L2/10/NCBiR/2011, permitting the experiments to be completed in Toronto, ON, Canada.

\section{REFERENCES}

Amin, R. M., Bhayana, B., Hamblin, M. R., and Dai, T. (2016). Antimicrobial blue light inactivation of Pseudomonas aeruginosa by photo-excitation of endogenous porphyrins: in vitro and in vivo studies. Lasers Surg. Med. 48, 562-568. doi: 10.1002/lsm.22474

Arenas, Y., Monro, S., Shi, G., Mandel, A., McFarland, S., and Lilge, L. (2013). Photodynamic inactivation of Staphylococcus aureus and methicillin-resistant Staphylococcus aureus with $\mathrm{Ru}(\mathrm{II})$-based type I/type II photosensitizers. Photodiagnosis Photodyn. Ther. 10, 615-625. doi: 10.1016/j.pdpdt.2013.07.001

Cassidy, C. M., Donnelly, R. F., and Tunney, M. M. (2010). Effect of sub-lethal challenge with Photodynamic Antimicrobial Chemotherapy (PACT) on the antibiotic susceptibility of clinical bacterial isolates. J. Photochem. Photobiol. B 99, 62-66. doi: 10.1016/j.jphotobiol.2010.02.004

Cockerill, F. R. III, Wilson, J. W., Vetter, E. A., Goodman, K. M., Torgerson, C. A., Harmsen, W. S., et al. (2004). Optimal testing parameters for blood cultures. Clin. Infect. Dis 38, 1724-1730. doi: 10.1086/421087

Dowsett, C. (2015). Breaking the cycle of hard-to-heal wounds: balancing cost and care. Wounds Int. 6, 17-21.

Ene, R., Panti, Z., Albu, E., Ene, P., Cirstoiu, M. M., and Cirstoiu, F. C. (2015). Negative pressure, a "Solution" in the treatment of infected knee prosthesis? Modica (Buchar) 10, 5-9.

Ervin, A. M., Wojciechowski, R., and Schein, O. (2010). Punctal occlusion for dry eye syndrome. Cochrane Database Syst. Rev. 9:CD006775. doi: 10.1002/14651858.CD006775.pub2

Fong, J., Kasimova, K., Arenas, Y., Kaspler, P., Lazic, S., Mandel, A., et al. (2015). A novel class of ruthenium-based photosensitizers effectively kills in vitro cancer cells and in vivo tumors. Photochem. Photobiol. Sci. 14, 2014-2023. doi: $10.1039 / \mathrm{c} 4 \mathrm{pp} 00438 \mathrm{~h}$

Halcon, L., and Milkus, K. (2004). Staphylococcus aureus and wounds: a review of tea tree oil as a promising antimicrobial. Am. J. Infect. Control 32, 402-408. doi: 10.1016/j.ajic.2003.12.008

Hamblin, M. R., Zahra, T., Contag, C. H., McManus, A. T., and Hasan, T. (2003). Optical monitoring and treatment of potentially lethal wound infections in vivo. J. Infect. Dis. 187, 1717-1725. doi: 10.1086/375244

Hashimoto, M. C., Prates, R. A., Kato, I. T., Nunez, S. C., Courrol, L. C., and Ribeiro, M. S. (2012). Antimicrobial photodynamic therapy on drug-resistant Pseudomonas aeruginosa-induced infection. An in vivo study. Photochem. Photobiol. 88, 590-595. doi: 10.1111/j.1751-1097.2012.01137.x

Jacques, S. L. (2013). Corrigendum: optical properties of biological tissues: a review. Phys. Med. Biol. 58, 5007-5008. doi: 10.1088/0031-9155/58/14/5007

Joubert, E., Gelderblom, W. C., Louw, A., and de Beer, D. (2008). South African herbal teas: Aspalathus linearis, Cyclopia spp. and Athrixia phylicoides-a review. J. Ethnopharmacol. 119, 376-412. doi: 10.1016/j.jep.2008.06.014

Kaspler, P., Lazic, S., Forward, S., Arenas, Y., Mandel, A., and Lilge, L. (2016). A ruthenium(ii) based photosensitizer and transferrin complexes enhance photo-physical properties, cell uptake, and photodynamic therapy safety and efficacy. Photochem. Photobiol. Sci. 15, 481-495. doi: 10.1039/c5pp00450k

Khan, A. A., Morrison, A., Hanley, D. A., Felsenberg, D., McCauley, L. K., O'Ryan, F., et al. (2015). Diagnosis and management of osteonecrosis of the jaw: a systematic review and international consensus. J. Bone Miner. Res. 30, 3-23. doi: 10.1002/jbmr.2405

Kirketerp-Møller, K., Zulkowski, K., and Garth, J. (2011). "Chronic wound colonization, infection, and biofilms," in Biofilm Infections, eds B. Thomas, Ø. J. Peter, M. Claus, and H. Niels (New York, NY: Springer-Verlag), 11-24.

Kucera, J., Sojka, M., Pavlik, V., Szuszkiewicz, K., Velebny, V., and Klein, P. (2014). Multispecies biofilm in an artificial wound bed-A novel model for in vitro assessment of solid antimicrobial dressings. J. Microbiol. Methods 103, 18-24. doi: 10.1016/j.mimet.2014.05.008

\section{ACKNOWLEDGMENTS}

Theralase Inc. provided the photosensitizer TLD1411 and the light sources for these studies. The authors appreciated Dr. Manjunatha Ankathatti Munegowda careful revision of the manuscript.

Kumar, M. S., Kirubanandan, S., Sripriya, R., and Sehgal, P. K. (2008). Triphala promotes healing of infected full-thickness dermal wound. J. Surg. Res. 144, 94-101. doi: 10.1016/j.jss.2007.02.049

Lee, P. C., and Rodgers, M. A. (1987). Laser flash photokinetic studies of rose bengal sensitized photodynamic interactions of nucleotides and DNA. Photochem. Photobiol. 45, 79-86. doi: 10.1111/j.1751-1097.1987.tb08407.x

Lee, S. Y., Seong, I. W., Kim, J. S., Cheon, K. A., Gu, S. H., Kim, H. H., et al. (2011). Enhancement of cutaneous immune response to bacterial infection after low-level light therapy with $1072 \mathrm{~nm}$ infrared light: a preliminary study. J. Photochem. Photobiol. B 105, 175-182. doi: 10.1016/j.jphotobiol.2011.08.009

Lilge, L., Tierney, K., and Nussbaum, E. (2000). Low-level laser therapy for wound healing: feasibility of wound dressing transillumination. J. Clin. Laser Med. Surg. 18, 235-240. doi: 10.1089/clm.2000.18.235

Mari, C., Pierroz, V., Rubbiani, R., Patra, M., Hess, J., Spingler, B., et al. (2014). DNA intercalating $\mathrm{Ru}(\mathrm{II})$ polypyridyl complexes as effective photosensitizers in photodynamic therapy. Chemistry 20, 14421-14436. doi: 10.1002/chem.201402796

Melo, W. C. M. A., Castro, L. F., Dal'Mas, R. M. M. T. S., and Perussi, J. R. (2011). "Effectiveness of photodynamic therapy on Gram-negative bacteria," in Science Against Microbial Pathogens: Communicating Current Research and Thechnological Advances, ed. A. Mendez-Vilas (Badajoz: Formatex Research Center), 662-667.

Murphy, P. S., and Evans, G. R. (2012). Advances in wound healing: a review of current wound healing products. Plast. Surg. Int. 2012:190436. doi: $10.1155 / 2012 / 190436$

Nussbaum, E. L., Lilge, L., and Mazzulli, T. (2003). Effects of low-level laser therapy (LLLT) of $810 \mathrm{~nm}$ upon in vitro growth of bacteria: relevance of irradiance and radiant exposure. J. Clin. Laser Med. Surg. 21, 283-290. doi: $10.1089 / 104454703322564497$

Nussbaum, E. L., Mazzulli, T., Pritzker, K. P., Heras, F. L., Jing, F., and Lilge, L. (2009). Effects of low intensity laser irradiation during healing of skin lesions in the rat. Lasers Surg. Med. 41, 372-381. doi: 10.1002/lsm.20769

Pardoll, D. M. (2012). The blockade of immune checkpoints in cancer immunotherapy. Nat. Rev. Cancer 12, 252-264. doi: 10.1038/nrc3239

Ragas, X., He, X., Agut, M., Roxo-Rosa, M., Gonsalves, A. R., Serra, A. C., et al. (2013). Singlet oxygen in antimicrobial photodynamic therapy: photosensitizerdependent production and decay in E. coli. Molecules 18, 2712-2725. doi: 10.3390/molecules18032712

Ramphal, R., Balloy, V., Jyot, J., Verma, A., Si-tahar, M., and Chignard, M. (2008). Recognition of either lipopolysaccharide or flagellin. J. Immunol. 181, 2-8. doi: 10.4049/jimmunol.181.1.586

Ravenscroft, M. J., Harker, J., and Buch, K. A. (2006). A prospective, randomised, controlled trial comparing wound dressings used in hip and knee surgery: Aquacel and Tegaderm versus Cutiplast. Ann. R. Coll. Surg. Engl. 88, 18-22. doi: 10.1308/003588406X82989

Reddi, E., Ceccon, M., Valduga, G., Jori, G., Bommer, J. C., Elisei, F., et al. (2002). Photophysical properties and antibacterial activity of meso-substituted cationic porphyrins. Photochem. Photobiol. 75, 462-470. doi: 10.1562/00318655(2002)075<0462:PPAAAO > 2.0.CO;2

Ronzani, F., Trivella, A., Arzoumanian, E., Blanc, S., Sarakha, M., Richard, C., et al. (2013). Comparison of the photophysical properties of three phenothiazine derivatives: transient detection and singlet oxygen production. Photochem. Photobiol. Sci. 12, 2160-2169. doi: 10.1039/c3pp50246e

Saito, A., Miyazaki, H., Fujie, T., Ohtsubo, S., Kinoshita, M., Saitoh, D., et al. (2012). Therapeutic efficacy of an antibiotic-loaded nanosheet in a murine burn-wound infection model. Acta Biomater. 8, 2932-2940. doi: 10.1016/j.actbio.2012.04.019

Schreier, R., and Chatterjee, A. (2015). Infections in the Immunocompromised Host. Medscape [Online]. Available at: http://emedicine.medscape.com/article/ 973120-overview\#showall [Accessed 05 Feb. 2016]. 
Schreml, S., Szeimies, R. M., Prantl, L., Karrer, S., Landthaler, M., and Babilas, P. (2010). Oxygen in acute and chronic wound healing. Br. J. Dermatol. 163, 257-268. doi: 10.1111/j.1365-2133.2010.09804.x

Sen, C. K., Gordillo, G. M., Roy, S., Kirsner, R., Lambert, L., Hunt, T. K., et al. (2009). Human skin wounds: a major and snowballing threat to public health and the economy. Wound Repair Regen. 17, 763-771. doi: 10.1111/j.1524475X.2009.00543.X

Shepard, J. R., Addison, R. M., Alexander, B. D., Della-Latta, P., Gherna, M., Haase, G., et al. (2008). Multicenter evaluation of the Candida albicans/Candida glabrata peptide nucleic acid fluorescent in situ hybridization method for simultaneous dual-color identification of C. albicans and C. glabrata directly from blood culture bottles. J. Clin. Microbiol. 46, 50-55. doi: 10.1128/JCM.01385-07

Shi, G., Monro, S., Hennigar, R., Colpitts, J., Fong, J., Kasimova, K., et al. (2015). $\mathrm{Ru}$ (II) dyads derived from $\alpha$-oligothiophenes: a new class of potent and versatile photosensitizers for PDT. Coord. Chem. Rev. 282-283, 127-138. doi: 10.1016/j.ccr.2014.04.012

Taraszkiewicz, A., Fila, G., Grinholc, M., and Nakonieczna, J. (2013). Innovative strategies to overcome biofilm resistance. Biomed. Res. Int. 2013:150653. doi: $10.1155 / 2013 / 150653$

Troxler, M., Vowden, K., and Vowden, P. (2006). Integrating Adjunctive Therapy into Practice: the Importance of Recognising 'hard-to-heal' Wounds. World Wide
Wounds. Available at: www.worldwidewounds.com/2006/december/Troxler/ Integrating-Adjunctive-Therapy-Into-Practice.html

Wong, T. W., Wang, Y. Y., Sheu, H. M., and Chuang, Y. C. (2005). Bactericidal effects of toluidine blue-mediated photodynamic action on Vibrio vulnificus. Antimicrob. Agents Chemother. 49, 895-902. doi: 10.1128/AAC.49.3.895902.2005

Zolfaghari, P. S., Packer, S., Singer, M., Nair, S. P., Bennett, J., Street, C., et al. (2009). In vivo killing of Staphylococcus aureus using a lightactivated antimicrobial agent. BMC Microbiol. 9:27. doi: 10.1186/14712180-9-27

Conflict of Interest Statement: The authors declare that the research was conducted in the absence of any commercial or financial relationships that could be construed as a potential conflict of interest.

Copyright ( 2016 Fila, Kasimova, Arenas, Nakonieczna, Grinholc, Bielawski and Lilge. This is an open-access article distributed under the terms of the Creative Commons Attribution License (CC BY). The use, distribution or reproduction in other forums is permitted, provided the original author(s) or licensor are credited and that the original publication in this journal is cited, in accordance with accepted academic practice. No use, distribution or reproduction is permitted which does not comply with these terms. 